\title{
A PROPOS DE SIMULIUM (PROSIMULIUM) HIRTIPES FRIES 1824 : \\ OBSERVATIONS MORPHOLOGIQUES ET BIOLOGIQUES STATIONS NOUVELLES POUR LA VARIÊTÉ ARVERNENSE GRENIER 1947
}

Par J.-M. DOBY et S. DEBLOCK

L'espèce type, décrite par Friès en 1824, d'après des exemplaires originaires de Norvège, présente, chez la nymphe, des filaments respiratoires au nombre de 16. Des variations importantes existent en ce qui concerne le nombre de ces filaments, puisque leur nombre peut atteindre 50 à 60 chez certains $S$. hirtipes, originaires d'Amérique du Nord (Malloch, 1914), où existe d'ailleurs également la forme à 16 filaments. Pour une forme caractérisée par un nombre de filaments variant de 25 à 30 , Grenier (1947) proposait la variété $S$. $(\boldsymbol{P}$.) hirtipes, var. arvernense.

\section{Observations morphologiques et biologiques}

Puri (1925) décrit le cocon de $S$. hirtipes comme une masse de soie tissée très grossièrement recouvrant totalement la nymphe, dont ne sont visibles que les filaments respiratoires. Partout où nous avons trouvé cette espèce (la Semoy dans les Ardennes, la Rosanna dans le Tyrol autrichien, la Sorède, la Baillaurie, la Massane dans les Pyrénées-Orientales), que nous ayons eu affaire à des exemplaires nymphaux à 16 ou à 25 filaments, nous n'avons que tout à fait exceptionnellement rencontré ce type de cocon. Celui-ci consistait au contraire le plus souvent en un simple feutrage globuleux, dans lequel était ancrée solidement l'extrémité postérieure de l'abdomen (1). Là du moins où nous l'avons trouvée, il semble que la nymphe de $S$. hirtipes n'est complètement protégée que lorsque la larve,

(1) Il est à remarquer que Edwards (1920), transcrivant les descriptions des auteurs américains concernant la forme à 50 filaments, dit \& pupae only partly covered ».

Ann. de Parastrologie, T. XXX, $\mathrm{N}^{\circ} 3 .-1955$. 
au moment de l'édification du cocon, a pu disposer de matériaux de complément. C'est ainsi que nous avons pu observer des cocons d'aspect très divers et si différents de ceux des autres espèces, si typiques en dépit de leurs formes pourtant variées, qu'ils pouvaient soit passer totalement inaperçus, soit être confondus, pour un œil non averti, avec les fourreaux de certains autres insectes torrenticoles (Trichoptères). La niche nymphale est alors formée par des grains de sable grossier, des paillettes de schistes, des débris végétaux maintenus ensemble par des fils de soie. Nous avons même eu la chance de pouvoir suivre en élevage l'édification de tels cocons et d'observer l'inclusion, dans les mailles de ceux-ci, de débris végétaux entrainés par le courant. Nous avons également rencontré des exemplaires nymphaux logés dans des fragments de limbe foliaire roulés en cornet et maintenus fermés par quelques fils de soie. Enfin, à plusieurs reprises, nous avons récolté des nymphes logées dans des cocons vides d'autres espèces ( $S$. ornatum dans la Semoy, S. monticola dans la Rosanna).

Il nous a paru intéressant de signaler ces faits en raison de la valeur systématique importante attribuée par la plupart des auteurs à la morphologie du cocon pour la détermination spécifique des nymphes.

Les observation biologiques que nous avons pu faire sur l'espèce type, dont la vie présente encore un certain nombre de points obscurs, corroborent celles effectuées par d'autres chercheurs. Il semble bien en effet que, pour cette espèce d'eau froide, il n'y ait qu'une seule génération annuelle, puisque, dans les Ardennes, nous l'avons trouvée à mi-avril 1953, sous forme nymphale à maturité (température de l'eau variant autour de $6^{\circ}$ C.), dans les Pyrénées, en avril 1954 (1) (température de l'eau entre 7 et $9^{\circ}$ C.), principalement au stade nymphal. Enfin, au Tyrol, cette année, si nous l'avons récoltée au mois d'août, encore au stade nymphal, il est à remarquer que la saison exceptionnellement froide avait, dans cette région, considérablement retardé le cycle normal de la faune et de la flore (température de l'eau entre 9 et $11^{\circ} \mathrm{C}$.).

\section{Présence de la variété arvernense Grenier 1947 dans les Pyrénées-Orientales}

C'est cette variété, rencontrée jusqu'ici exclusivement en France, dans le Massif Central, que nous avons eu l'occasion de récolter

(1) En été, elle n'existait ni au stade larvaire, ni au stade nymphal.

ANN. de Parastrologie, T. XXX, N ${ }^{\circ} 3 .-195$.

17. 
dans les Pyrénées-Orientales. Tous les exemplaires présentaient régulièrement 25 filaments de chaque côté.

Du moins en ce qui concerne l'année 1954, nous l'avons trouvée, à deux reprises, dans des petits cours d'eau à lit rocheux dans le Massif des Albères. Dans la Sorède (altitude $300 \mathrm{~m}$.), la variété à 25 filaments et celle à 16 coexistaient, dans la proportion de une de la variété arvernense (1) pour deux de l'espèce type. Nous n'avons observé que la première variété dans la Massane (altitude $700 \mathrm{~m}$.) et la seconde seulement dans la Baillaurie (altitude $125 \mathrm{~m}$.). L'état de maturité des nymphes et le nombre d'enveloppes nymphales vides permettent de situer l'éclosion des adultes, aussi bien pour l'une que pour l'autre des deux variétés, aux environs du 15 avril pour la Sorède et du 25 avril pour la Massane, dont la vallée est plus froide en raison de son altitude et de son orientation. Dans les deux stations de la variété arvernense vivaient également, à l'état larvaire ou nymphal : $S$. aureum Fries 1824, $S$. monticola Friedederichs 1920, $S$. variegatum Meigen 1818 et $S$. latipes Meigen 1804 (2).

Des formes adultes du genre Prosimulium, différant principalement de l'espèce hirtipes par une coloration générale plus claire, ont été trouvées à plusieurs reprises dans les Pyrénées, notamment à Thues (Walsingham) (3), à Vernet (Lesné) (3), à Massey (Pandellé) (3), et dans les Hautes-Pyrénées (Surcouf) (3). Parmi celles-ci et parmi d'autres, également plus claires, capturées dans le Massif Central, certaines, déterminées $S$. rufipes Meigen $1830(=S$. fulvipes Edwards 1921) et $S$. gallii Edwards 1921, sont considérées par Edwards comme des formes méridionales de S. hirtipes. Selon lui, elles correspondraient peut-être aux formes nymphales à 25 filaments, dont Grenier a fait la variété arvernense. Toutefois, jusque maintenant, $S$. hirtipes var. arvernense n'était connue que du seul Massif Central.

Son existence dans les Pyrénées-Orientales semblerait donc appuyer l'hypothèse de cet auteur. Le problème eût été résolu d'une façon définitive par la découverte d'adultes. Malheureusement, nous n'avons pas rencontré d'adultes du genre Prosimulium aux environs immédiats des deux stations précitées, et les conditions de notre récolte ne nous ont pas permis d'obtenir l'éclosion au laboratoire des nymphes récoltées.

(1) M. Grenier nous a dit l'avoir récemment rencontrée également dans du matériel en provenance d'Espagne (observation non publiée).

(2) Nous avons recherché en vain S. hirtipes var. arvernense dans d'abondantes récoltes nymphales en provenance de divers torrents du Tyrol et des Ardennes, alors que l'espèce type s'y trouve en abondance.

(3) in Séguy, 1925. 

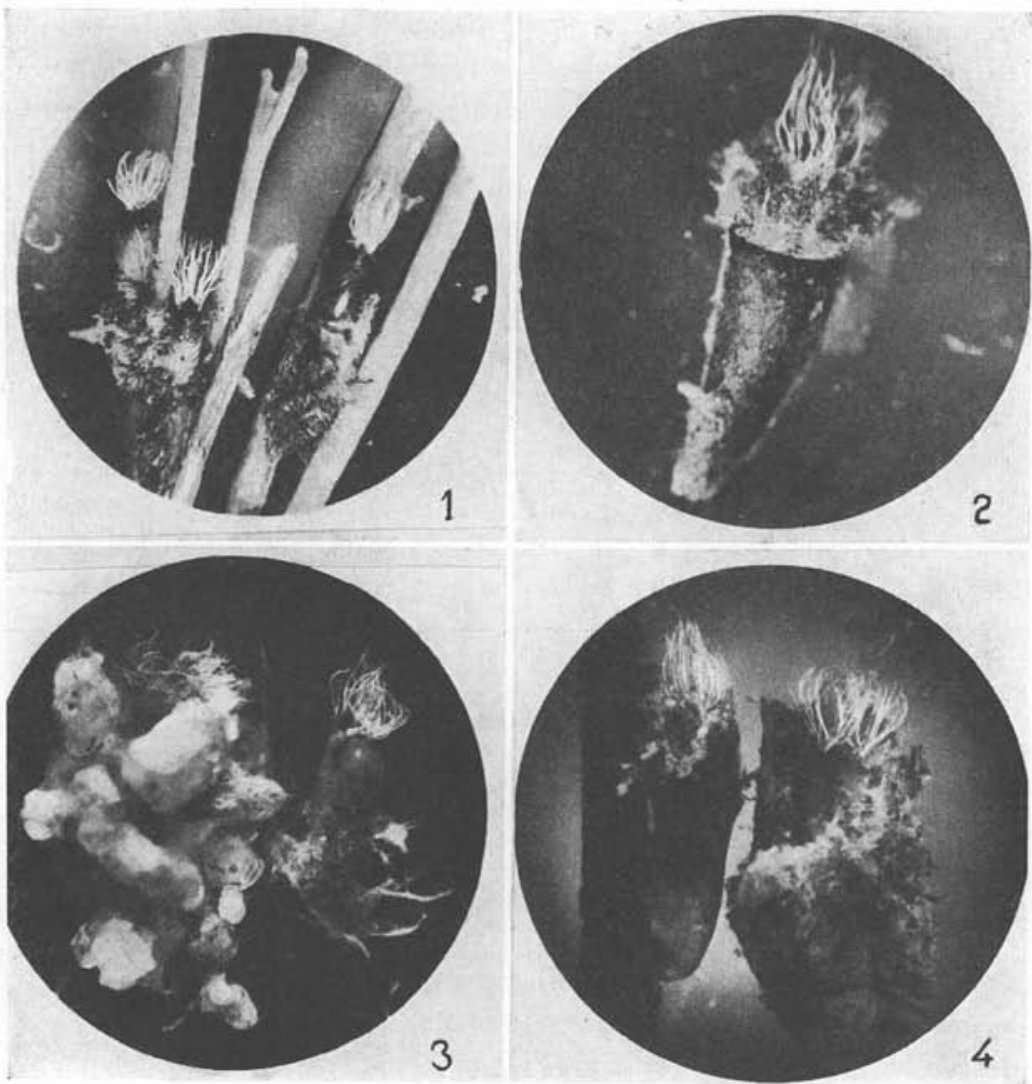

Fic. 1. - S. hirtipes type de la Semoy (Ardennes). Exemplaires nymphaux à cocons réduits.

Fra. 2. - S. hirtipes type de la Semoy (Ardennes). Exemplaire nymphal logé dans un cocon vide de $S$. ornatum.

Fic. 3. - A gauche: S. hirtipes var. arvernense de la Massane (Pyr.-Orient.), dans un cocon formé de sable grossier (un grain volumineux a été détaché pour permettre une vue meilleure des filaments).

A droite : $S$. hirtipes type de la Baillaurie (Pyr--Orient.), nymphe d'élevage avec débris végétaux nombreux inclus dans les mailles du cocon.

Fig. 4. - A gauche : S. hirtipes type de la Rosanna (Tyrol autrichien). Exemplaire nymphal logé dans un cocon vide de $S$. monticola.

A droite : S. hirtipes type de la Rosanna (Tyrol autrichien). Exemplaire nymphal logé dans un morceau de limbe foliaire roulé en cornet. (Un fragment de cclui-ci a été découpé. Seule était visible l'extrémité des filaments). 
Par ailleurs, la validité de $S$. rufipes et de $S$. gallii a été fortement mise en doute par Grenier (1947), en raison des différences de mélanisme qui apparaissent dans les descriptions de $S$. hirtipes faites par les divers auteurs, en raison également de l'identité des divers caractères morphologiques (genitalia notamment) d'exemplaires de $S$. hirtipes, $S$. gallii, $S$. rufipes examinés par lui. Nous-mêmes, étudiant deux lots de femelles du genre Prosimulium, avons pu également constater d'importantes différences dans le mélanisme, et cela même parmi des exemplaires d'origine identique. Un lot (30 \&), cap-

\begin{tabular}{|c|c|c|c|c|}
\hline Caractìnes & $\begin{array}{l}\text { S. rufipes } \\
\text { EXEMPLAIRES } \\
\text { DU MUSÉUM }\end{array}$ & $\begin{array}{l}\text { S. hirtipes } \\
\text { Exemplaires } \\
\text { DU CANigou }\end{array}$ & $\begin{array}{l}\text { S. hirtipes } \\
\text { Exemplarres } \\
\text { DU TYROL. }\end{array}$ & $\begin{array}{l}\text { S. hirtipes } \\
\text { DESCRIPTION } \\
\text { EDWARDS } \\
\text { D'APRÈS DES } \\
\text { EXEMPLAIRES } \\
\text { D'ÉCOSSE }\end{array}$ \\
\hline $\begin{array}{c}\text { Fémur (patte } \\
\text { médiane).... }\end{array}$ & Jaune & $\begin{array}{c}\text { Jaune foncé deve- } \\
\text { nant noiratre à } \\
\text { partir du } 1 / 4 \\
\text { distal sur la face } \\
\text { externe }\end{array}$ & $\begin{array}{c}\text { Brun jaune foncé, } \\
\text { devenant noir } \\
\text { à partir du } 1 / 2 \\
\text { distal sur la } \\
\text { face externe }\end{array}$ & $\begin{array}{c}\text { ¿ Pattes } \\
\text { entièrement } \\
\text { sombres } b\end{array}$ \\
\hline Tibia.......... & $\begin{array}{l}\text { Jaune deve- } \\
\text { nant brun sur } \\
\text { le } 1 / 5 \text { distal }\end{array}$ & $\begin{array}{l}\text { Jaune foncé, } \\
\text { noirâtre sur les } \\
1 / 5 \text { proximal } \\
\text { et distal (1) }\end{array}$ & $\begin{array}{c}\text { Brun jaune foncé, } \\
\text { devenant noir sur } \\
\text { le } 1 / 5 \text { proximal } \\
\text { et à partir } \\
\text { du } 1 / 2 \text { distal }\end{array}$ & \\
\hline Basitarse...... & $\begin{array}{l}\text { Brun devenant } \\
\text { foncé à sa } \\
\text { partie distale }\end{array}$ & Brun foncé & Noirâtre & \\
\hline Tarse ......... & Brun foncé & Brun noirâtre & Noirâtre & \\
\hline $\begin{array}{c}\text { Pilosité du } \\
\text { thorax ..... }\end{array}$ & Jaune doré & Jaune pâle & Jaune pâle & $\begin{array}{c}\text { " Jaunâtre } \\
\text { pâle } ~\end{array}$ \\
\hline $\begin{array}{l}\text { Pilosité de } \\
\text { l'abdomen.. }\end{array}$ & $\begin{array}{l}\text { Jaune doré } \\
\text { Soies longues }\end{array}$ & $\begin{array}{l}\text { Jaune pâle } \\
\text { Soies moyennes }\end{array}$ & $\begin{array}{c}\text { Jaune pâle } \\
\text { Soies moyennes }\end{array}$ & \\
\hline $\begin{array}{r}\text { Pilosité des } \\
\text { pattes....... }\end{array}$ & Jaune doré & Jaunâtre & Jaune foncé & \\
\hline
\end{tabular}

(1) Le noircissement des extrémités distale du fémur et proximale du tibia donne un aspect géniculé net aux exemplaires du Canigou. Ce \& genou »sombre n'est pratiquement pas visible chez l'exemplaire de $S$. rufipes. Les caractères donnés pour les exemplaires du Canigou correspondent à un type moyen. Dans ce lot $(30$ ) $)$ existe en effet une certaine gradation dans l'intensité du mélanisme. 
turé dans le Massif du Canigou (1), où nous n'avons pu trouver de nymphes, ni à 16 , ni à 25 filaments, semble présenter des caractères de coloration intermédiaires entre ceux d'un lot de $S$. hirtipes (8 $q$ ) récolté par nous dans le Tyrol autrichien, où n'existe que la forme à 16 filaments, et un exemplaire de $S$. rufipes (collection du $\mathrm{Mu}$ séum, détermination Séguy) (2). Si le lot en provenance du Tyrol se montre sensiblement homogène, celui du Canigou par contre présente certaines variations dans l'intensité de la teinte. L'étude comparative de ce matériel est donnée dans le tableau ci-joint. Ces variations semblent bien confirmer les conclusions de Grenier en ce qui concerne la validité des espèces $S$. rufipes et $S$. gallii.

\section{BIBLIOGRAPHIE}

Edwards (F. W.). - On the british species of Simulium. I. The adults. Bull. of Entomol. Res., VI, 1915, p. 23.

Edwards (F. W.). - On the british species of Simulium. II. The early stages ; with corrections and additions to part I. Bull. of Entomol. Res., XI, 1920, p. 211.

Grenier (P.). - Notes morphologiques et biologiques sur quelques simulies nouvelles pour la faune française. Bull. Soc. ent. France, LII, 1947, p. 66.

Grenier (P.). - Simuliidw de France et d'Afrique du Nord. Encyclopédie Entomologique, Lechevalier éd., Paris, 1953.

MaLloch (J. R.). - American black-flies or buffalognats. U.S. Dept. of agric., Bureau of Entom., $\mathrm{n}^{\circ}$ 26, in PURI, 1925.

PUni (I. M.). - On the life history and structure of the early stages of Simuliida (Diptera, Nematocera). Part II. Parasitology, XVII, 1925, p. 335.

Sḱguy (E.). - Ptychopteride, Orphnephilide, Simuliidæ, Culicide, Psychodidæ Phlebotomina. Faune de France, Lechevalier éd., Paris, 1925.

Laboratoire Arago de Banyuls (Directeur: M. le Professeur Petrit) et Laboratoire de Parasitologie et Zoologie médicale de la Faculté de Médecine et Pharmacie de Lille (Directeur : M. le Professeur Coutelen).

(1) Le Massif du Canigou est situé à $45 \mathrm{~km}$. à vol d'oiseau du Massif des Albères.

(2) Il ne nous est malheureusement pas possible de connaitre l'origine de cet exemplaire qui correspond toutefois en tous points à la description de $S$. rufipes Meigen, 1830. 